\title{
The Fragmentation Region of High Energy Nucleus-Nucleus and Hadron-Nucleus Collisions
}

\author{
Larry McLerran ${ }^{1, \star}$ \\ ${ }^{1}$ Institute for Nuclear Theory, University of Washington, Physics/Astronomy Bldg. Box 351550, Seattle WA \\ 98195-1550
}

\begin{abstract}
The fragmentation region of particles produced in high energy nuclear collisions provides a laboratory for studying high baryon number density systems. This talk outlines work in progress that attempts to compute properties of the matter produced in these collisions at the highest energies.
\end{abstract}

\section{Introduction}

The earliest studies of the production of the Quark Gluon Plasma in ultra-relativistic heavy ions collisions concerned the fragmentation region[1]. The energy considered was high enough so that the fragmentation regions of the two nuclei would be separated. This requirement is easiest to understand in the rest frame of one of the target nucleus. A produced fragment of the projectile with a gamma factor $\gamma$ and a formation time $t_{\text {for }}$ must be produced outside the target,

$$
\gamma t_{\text {for }}>>R
$$

Using a formation time of order the inverse transverse mass, this becomes

$$
E / M_{T}^{2}>>R
$$

For collisions with energies of the order of 10's to 100's of GeV, a typical transverse mass is a few hundred $\mathrm{MeV}$, and we see that for a large nucleus such as gold this is satisfied if the projectile has an energy larger than about $15 \mathrm{GeV}$, corresponding to a center of mass energy of the order of $30 \mathrm{GeV}$ per nuclei. This is the energy range where a central region of low baryon density begins to emerge.

In these early estimates, the energy densities were estimated in the fragmentation region to be a few $\mathrm{GeV} / \mathrm{Fm}^{3}$, baryon densities several times that of nuclear matter and lifetimes of order the produced matter of order $10 \mathrm{Fm} / \mathrm{c}$. It was argued that similar energy densities and lifetimes might be made in the central region[1]. Bjorken then developed an elegant and simple picture for the central region[2], and formulated hydrodynamic equations for the description of thermalized matter produced in ultrarelativistic heavy ion collisions. These developments led to a standard picture of heavy ion collisions which is illustrated ibn Fig. 1.

There is now a consistent space-time picture that descirbes the thermalized matter formed in the central region of heavy ion collisions. This picture uses the color charges associated with the Color

\footnotetext{
^e-mail: 1mcler@uw.edu
} 


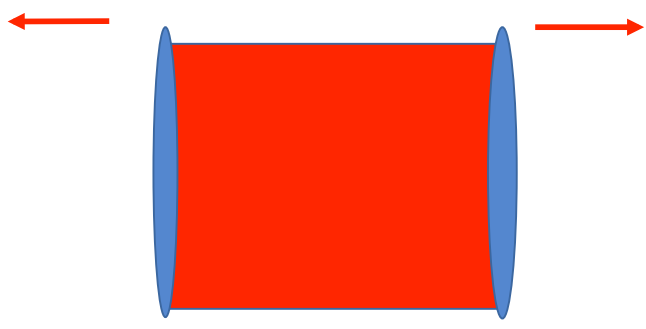

Figure 1. A conceptual picture for the production of matter in ultra-relativistic heavy ion collisions.

Glass Condensate[3]-[4] of the heavy nuclei to produce highly coherent color electric and magnetic fields, the Glasma[5]-[6], that ultimately evolves into a thermalized Quark Gluon Plasma[7]-[8].

What I will present in the talk is an outline of work which I am doing with Srimoyee Sen and Soeren Schlicting, to generalize the computation of the initial distribution of matter in the central region to the baryon rich fragmentation region[9]. This has an extra complication relative to the Glasma computation, since one must determine the evolution of the distribution of baryon matter, in addition to computing the formed color fields and their gluon radiation.

What I will consider in this talk is the fragmentation region produced in collisions at asymptotically high energies. In principle, these considerations might be extended to lower energies where the fragmentation regions do not fully separate but where the high energy description of the collision is valid. This problem is more complicated and we do not consider it here.

In Fig. 2, I show the distribution of baryons and of mesons as seen in a high energy collision. The fragmentation region is the region of a few units of rapidity around that of the prjectile and target where the baryon number is concentrated.

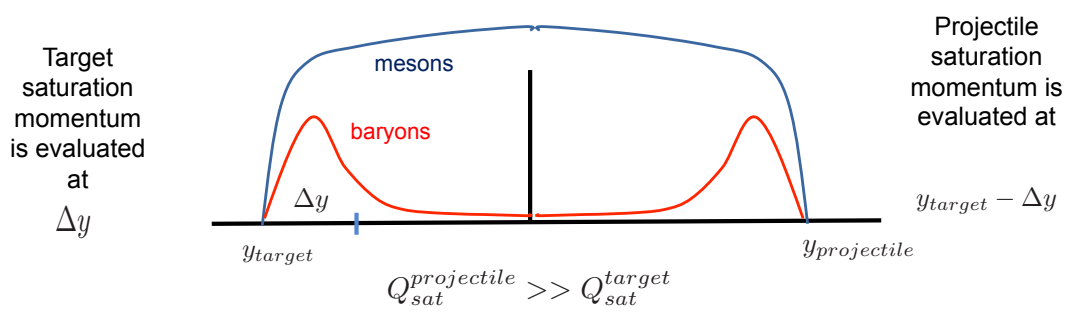

Figure 2. The rapidity distribution of particles and the fragmentation regions for ultra-relativistic nuclear collisions.

\section{Asymmetric Saturation Momenta of the Target and Projectile in the Fragmentation Region.}

Consider the kinematic region of the target. We expect the saturation momentum of the target to be of order

$$
Q_{\text {target }}^{\text {sat } 2} \sim A^{1 / 3} \Lambda_{Q C D}^{2} e^{\kappa \Delta y}
$$


where $\Delta y$ is the width of the fragmentation region and $\kappa$ is a constant of order $\kappa \sim .2-.3$. On the other hand, the saturation momentum of the projectile is much larger since it is evolved in rapidity from the rapidity of the projectile to that of the target

$$
Q_{\text {proj }}^{\text {sat } 2} \sim A^{1 / 3} \Lambda_{Q C D}^{2} e^{\kappa\left(y_{\text {prj }}-y_{\text {target }}\right)}
$$

At LHC energies, the target saturation momenta is of the order of a $\mathrm{GeV}$ but the projectile is of order $5-10 \mathrm{GeV}$. This means the projectile is black to the partons in the target up to a scale of momentum which is the projectile saturation momentum. The dominant particle production occurs in the region of momentum between these two saturation momenta. This is a region where the color sources produce a weak field $\mathrm{A} \ll 1 / \mathrm{g}$ and there is not much interaction of produced particles in this kinematic region, at least when the degrees of freedom correspond to classical fields. At momenta scales less than the saturation momenta of the target, there are strong fields and classical time evolution of classical fields. This latter region is that of the Glasma.

We can estimate the transverse momentum distribution of particles in various transverse momentum intervals[10]-[11]. For transverse momenta scales less than that of the target saturation momenta, the distribution will go to a constant,

$$
\frac{d N}{d y d^{2} p_{T}} \sim \text { cons, } p_{T}<Q_{\text {target }}^{\text {sat }}
$$

In the region of transverse momentum between the two scales, the produced gluons reflects the bremstrahlung distribution of the target gluons

$$
\frac{d N}{d y d^{2} p_{T}} \sim \frac{Q_{\text {target }}^{\text {sat }}}{p_{T}^{2}}, \quad Q_{\text {target }}^{\text {sat }}<p_{T}<Q_{\text {proj }}^{\text {sat }}
$$

For large transverse momenta, the distribution ahs the well known $1 / p_{T}^{4}$ particle production cross section

$$
\frac{d N}{d y d^{2} p_{T}} \sim \frac{Q_{\text {target }}^{2} Q_{\text {proj }}^{2}}{p_{T}^{4}} Q_{\text {proj }}^{\text {sat }}<p_{T}
$$

These transverse momentum distributions have consequences for the average transverse momentum and the multiplicity. Up to slowly growing terms, the total multiplicity is not affected by the increase of the projectile saturation momentum,

$$
\frac{d N}{d y} \sim Q_{\text {target }}^{2}
$$

On the other hand the transverse momentum per particle is greatly enhanced and is proportional to the saturation momentum of the projectile

$$
<p_{T}^{2}>\sim Q_{\text {proj }}^{2}
$$

The average transverse momentum per particle in the fragmentation region should increase by a factor of 100 between RHIC and LHC energies. Of course this is tempered somewhat by the fragmentation of the initially produced gluons. 


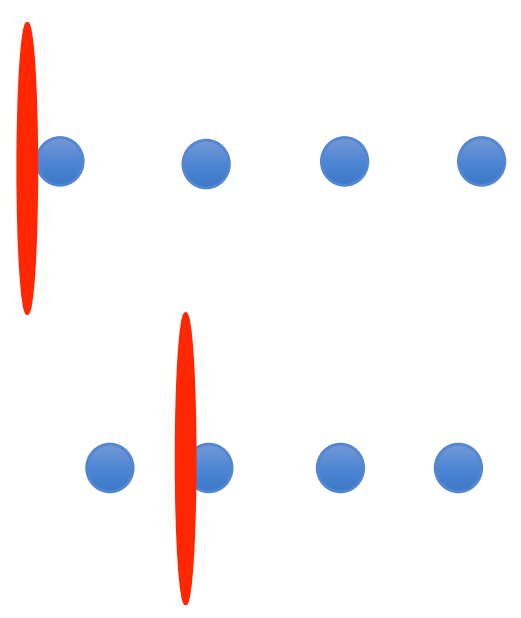

Figure 3. The sequential collision of a projectile nucleus with target baryons.

\section{Compression of Baryon Number}

Due to the fact that the target nucleons are hit sequentially by the Lorentz contracted projectile, there is compression of the target baryon number. This sequential collision is shown in Fig. 3. Due to the sequential nature of the collision, the nuclear separation is reduced by $\Delta z / z=1-v$ where $v$ is the velocity of the struck projectile baryon[1]. Some of this compression is artificial since it is associated with a moving distribution of baryons, and some of the apparent compression is simply Lorentz contraction. This reduces the compression by a gamma factors of the struck baryon, so that the physically relevant compression is

$$
\Delta z / Z \sim 1 / \gamma_{\text {baryon }}
$$

We can estimate the typical gamma factor. In the collision, as on mass shell target baryon passes through the projectile sheet. It acquires a transverse momentum of order $Q_{\text {proj }}^{s a t}$. The longitudinal $p^{-}$ of the baryon is unchanged since the projectile field is $x^{+}$independent. Therefore, the typical change in $p^{+}$is $p^{+} \sim Q_{\text {proj }}^{\text {sat } 2} / p^{-}$. This means there is a typical induced rapidity of order

$$
\Delta y \sim \frac{1}{2} \ln \left(p^{+} / p^{-}\right) \sim \ln \left(Q_{\text {sat }}^{\text {proj }} / m\right)
$$

corresponding to a Lorentz gamma factor fo $Q_{s a t}^{p r o j} / M$. We will take $M$ to be a typical momentum scale for the quark constituents of the nucleus when the nucleus is at rest,

The typical baryon number density after the collision is therefore of order

$$
N_{B} / V \sim Q_{\text {sat }}^{\text {proj }} \rho_{\text {nucleus }} / M
$$

For gluons when they are initially produced, their typical longitudinal size scale is also of order the saturation momentum. Therefore the gluon density and the compressed baryon density have the same dependence upon $Q_{\text {sat }}^{\text {proj }}$ Therefore

$$
N_{B} / N_{\text {gluon }} \sim \text { cons }
$$

that is indepdnent of beam energy. 
Now of course the expansion dynamics might change this result. Naively, this may not be such a large effect, because the dominant gluon production is in the region of weak field strength. To a first approximation, the multiplicity of these gluons might not change so much. Nevertheless, there may be a some partial dilution of this factor due to final state interaction. A measure of this quantity as a function of energy is therefore a probe of the importance of final state interactions and thermalization in the fragmentation region.

\section{The Ba-Glasma}

We shall refer to this baryon rich matter produced in the fragmentation region of the collision of two ultra-relativistic nuclei as the Ba-Glasma to differentiate it form the Glasma of the low baryon number density region. To set up the problem of computation for the baryon density and the gluon density in the collision from first principles in QCD is not so difficult. For the baryons, we can take a distribution of quarks in the initial state. We let them collide with a sheet of projectile nucleus color charge. This is a simple external field problem, and can be solved for the resulting baryon density.

Once the baryon density of the target is specified and the color field of the projectile assumed to be known, one can compute the distribution for produced gluons in the region that dominates the multiplicity, $Q_{t a r g}^{s a t}<p_{T}<Q_{\text {proj }}^{\text {sat }}$. In this region the field is weak and can be computed to lowest order in the strength of the target color field but all order in the projectile source strength. This is presumably possible because the projectile source strength is a delta function and should generate a field that is a gauge rotation of a small fluctuation field induced by the target source strength.

\section{Acknowledgements}

I thank my colleagues Soeren Schlichting and Srimoyee Sen with whom work is progressing along the lines outlined in this talk L. McLerran is supported under Department of Energy under grant number DOE grant No. DE-FG02-00ER41132.

\section{References}

[1] R. Anishetty, P. Koehler and L. D. McLerran, Phys. Rev. D 22, 2793 (1980). doi:10.1103/PhysRevD.22.2793

[2] J. D. Bjorken, Phys. Rev. D 27, 140 (1983). doi:10.1103/PhysRevD.27.140

[3] L. D. McLerran and R. Venugopalan, Phys. Rev. D 49 (1994) 2233 doi:10.1103/PhysRevD.49.2233 [hep-ph/9309289].

[4] L. D. McLerran and R. Venugopalan, Phys. Rev. D 49 (1994) 3352 doi:10.1103/PhysRevD.49.3352 [hep-ph/9311205].

[5] A. Kovner, L. D. McLerran and H. Weigert, Phys. Rev. D 52 (1995) 6231 doi:10.1103/PhysRevD.52.6231 [hep-ph/9502289].

[6] A. Kovner, L. D. McLerran and H. Weigert, Phys. Rev. D 52 (1995) 3809 doi:10.1103/PhysRevD.52.3809 [hep-ph/9505320].

[7] T. Epelbaum and F. Gelis, Phys. Rev. Lett. $111 \quad$ (2013) 232301 doi:10.1103/PhysRevLett.111.232301 [arXiv:1307.2214 [hep-ph]].

[8] J. Berges, B. Schenke, S. Schlichting and R. Venugopalan, Nucl. Phys. A 931 (2014) 348 doi:10.1016/j.nuclphysa.2014.08.103 [arXiv:1409.1638 [hep-ph]].

[9] L. McLerran, S. Sen and S. Schlichting, work in progress. 
[10] Y. V. Kovchegov and A. H. Mueller, Nucl. Phys. B 529 (1998) 451 doi:10.1016/S05503213(98)00384-8 [hep-ph/9802440].

[11] A. Dumitru and L. D. McLerran, Nucl. Phys. A 700 (2002) 492 doi:10.1016/S03759474(01)01301-X [hep-ph/0105268]. 